\title{
Boosting the Restoring Performance of Deduplication Data by Classifying Backup Metadata
}

\author{
RU YANG, Department of Computer Science, Jinan University, P. R.China \\ YUHUI DENG, Department of Computer Science, Jinan University, State Key Laboratory of Computer \\ Architecture, Institute of Computing Technology, Chinese Academy of Sciences, P. R.China \\ YI ZHOU, TSYS School of Computer Science, Columbus State University, USA \\ PING HUANG, College of Engineering, Temple University, USA
}

\begin{abstract}
Restoring data is the main purpose of data backup in storage systems. The fragmentation issue, caused by physically scattering logically continuous data across a variety of disk locations, poses a negative impact on the restoring performance of a deduplication system. Rewriting algorithms are used to alleviate the fragmentation problem by improving the restoring speed of a deduplication system. However, rewriting methods give birth to a big sacrifice in terms of deduplication ratio, leading to a huge storage space waste. Furthermore, traditional backup approaches treat file metadata and chunk metadata as the same, which causes frequent on-disk metadata accesses.

In this article, we start by analyzing storage characteristics of backup metadata. An intriguing finding shows that with 10 million files, the file metadata merely takes up approximately $340 \mathrm{MB}$. Motivated by this finding, we propose a Classified-Metadata based Restoring method (CMR) that classifies backup metadata into file metadata and chunk metadata. Because the file metadata merely takes up a meager amount of space, CMR maintains all file metadata in memory, whereas chunk metadata are aggressively prefetched to memory in a greedy manner. A deduplication system with CMR in place exhibits three salient features: (i) It avoids rewriting algorithms' additional overhead by reducing the number of disk reads in a restoring process, (ii) it increases the restoring throughput without sacrificing the deduplication ratio, and (iii) it thoroughly leverages the hardware resources to boost the restoring performance. To quantitatively evaluate the performance of CMR, we compare our CMR against two state-of-the-art approaches, namely, a history-aware rewriting method (HAR) and a context-based rewriting scheme (CAP). The experimental results show that compared to HAR and CAP, CMR reduces the restoring time by $27.2 \%$ and $29.3 \%$, respectively. Moreover, the deduplication ratio is improved by $1.91 \%$ and $4.36 \%$, respectively.
\end{abstract}

This work is sponsored by the National Natural Science Foundation of China under Grant No. 62072214 and Grant No. 61572232, the International Cooperation Project of Guangdong Province under Grant No. 2020A0505100040, and in part by the Open Research Fund of Key Laboratory of Computer System and Architecture, Institute of Computing Technology, Chinese Academy of Sciences under Grant (CARCH201705).

Authors' addresses: R. Yang, Department of Computer Science, Jinan University, Guangzhou, P. R. China, 510632; email: yangru.ay@foxmail.com; Y. Deng (corresponding author), Department of Computer Science, Jinan University; State Key Laboratory of Computer Architecture, Institute of Computing Technology, Chinese Academy of Sciences, P. R.China., 510632; email: tyhdeng@jnu.edu.cn; Y. Zhou, TSYS School of Computer Science, Columbus State University, Doimukh, USA; email: zhou_yi@columbusstate.edu; P. Huang, College of Engineering, Temple University, USA; email: templestorager @temple.edu.

Permission to make digital or hard copies of all or part of this work for personal or classroom use is granted without fee provided that copies are not made or distributed for profit or commercial advantage and that copies bear this notice and the full citation on the first page. Copyrights for components of this work owned by others than the author(s) must be honored. Abstracting with credit is permitted. To copy otherwise, or republish, to post on servers or to redistribute to lists, requires prior specific permission and/or a fee. Request permissions from permissions@acm.org.

(c) 2021 Copyright held by the owner/author(s). Publication rights licensed to ACM.

2577-3224/2021/04-ART13 \$15.00

https://doi.org/10.1145/3437261

ACM/IMS Transactions on Data Science, Vol. 2, No. 2, Article 13. Publication date: April 2021. 
CCS Concepts: • Computer systems organization $\rightarrow$ Embedded systems; Redundancy; Robotics; • Networks $\rightarrow$ Network reliability;

Additional Key Words and Phrases: Data deduplication, metadata, data backup, data restoring, fragmentation

ACM Reference format:

Ru Yang, Yuhui Deng, Yi Zhou, and Ping Huang. 2021. Boosting the Restoring Performance of Deduplication Data by Classifying Backup Metadata. ACM/IMS Trans. Data Sci. 2, 2, Article 13 (April 2021), 16 pages.

https://doi.org/10.1145/3437261

\section{INTRODUCTION}

With the advent of big data, the rapid growth of data volume poses a huge challenge to the limited storage space in data centers. Data deduplication greatly reduces the storage requirements for storing data and the bandwidth required for network transmission. It is becoming a key data reduction technique for modern backup systems. The purpose of storing data in a backup system is to restore data promptly. If an enterprise cannot recover data in a timely manner when a disk fails or a database-related file becomes corrupted, then the loss will be immeasurable [23]. Similarly, when a company in an emergency needs to recover historical data in a short period of time, recovery performance is critical to business success. Any increased latency can cause a massive loss to an enterprise [27]. For example, a 100-ms increase in latency will reduce Amazon's sale by $1 \%$, and an extra $0.5 \mathrm{~s}$ in search page display time can cut down revenues of Google by $20 \%$ [13]. However, any decreased latency can bring huge benefits to these enterprises. Hamilton et al. [11] suggest that an acceleration of just $5 \mathrm{~s}$ at Shopzill will result in a $25 \%$ increase in page views and a $10 \%$ increase in taxes, while the amount of hardware is reduced by $50 \%$ and Google's traffic is reduced by $120 \%$. Therefore, reducing the restoring time of backup systems becomes a vital factor for the enterprises that store their data within backups [30]. Disastrous recovery and quick access to historical backup data make the data recovery process increasingly critical.

Although a number of the deduplication technologies have been proposed for data backup [1, $6,14,16,31]$, they overlooked one key issue: fragmentation. Chunk fragmentation is caused by a chunk that is shared among multiple backups. Because the deduplication process eliminates redundant data chunks and stores only unique first instance of these data chunks, after deduplication of the latest backup data, the blocks of data required to restore the backup are physically scattered among previously stored backups. With the increase of data backups, the occurrence of chunk fragments seriously affects system recovery performance [25]. Although recovery performance can be improved by replacing magnetic tapes with magnetic disks, performance degradation of a recovery process cannot be avoided due to the deduplication fragmentation [5, 7]. Lillibridge et al. [15] demonstrated that fragmentation significantly degrades the performance of deduplication systems. The fragmentation becomes more severe as the number of backups increases. More specifically, the impact on the latest backup becomes particularly significant as more chunks exist in the previous backups.

To alleviate the impact of fragmentation, a variety of rewriting-based algorithms has been studied to improve the recovery performance of a backup system [12, 21, 24]. Rewrite algorithms take advantage of a common strategy, that is, treating an existing copy of a block as a new block and rewriting this block to improve recovery performance. Fu et al. [9] point out that a large number of existing rewriting methods (container capping rewriting) only maintain a fraction of the total backup stream, such a fractional backup stream maintenance dampens the recovery performance. Fu et al. [9] proposed a history-aware rewriting algorithm to reduce the unnecessary fragmentation rewriting. However, Fu's experimental results show that their history-aware rewriting loses nearly half of the deduplication ratio to improve restoring performance. 


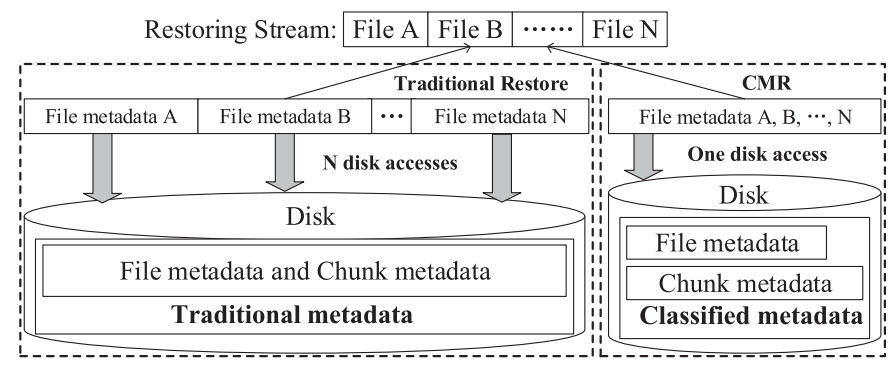

Fig. 1. Number of disk accesses with traditional restore and CMR.

A deduplication system generates a piece of metadata for a backup after the backup is completed so that the data can be restored later $[8,18,19,26]$. The metadata record some information about its backup, such as the logical order of the files in the backup, the size of files, the fingerprints of data blocks, and their locations on the disk. In our research, we classify the logical order of the files and the size of the files as file metadata and the information regarding the blocks (i.e., fingerprint, disk location, etc.) as chunk metadata. Therefore, in traditional deduplication systems, backup metadata are considered a combination of file metadata and chunk metadata. When a traditional system recovers data, it reads its file metadata and chunk metadata in order; in other words, it reads the current metadata first and then the subsequent metadata. These sequential reading approaches cannot read metadata at the same time, thereby giving rise to frequent access to on-disk metadata, damaging the recovery performance.

Meister et al. [18] suggest that most existing deduplication systems ignore the management and organization of backup metadata. To avoid extra overhead caused by rewriting algorithms and reduce the number of disk reads during the restoring process, we proposed a Classified Metadatabased Restoring (CMR) system that classifies the backup metadata into file metadata and chunk metadata. We analyzed the size of file metadata and found that for 10 million files, the file metadata are merely about $340 \mathrm{MB}$. This finding coupled with our metadata classification strategy motivated us to judiciously keep the entire file metadata, which takes up a meager space, within main memory and to aggressively prefetch chunk metadata from disk drives into memory. These two effects together significantly reduce the number of on-disk access for metadata. Unlike our CMR, traditional deduplication systems treat file metadata and chunk metadata as a whole. Because traditional deduplication systems are in lack of metadata classification ability, such systems have to access the whole metadata in a sequential manner, which results in excessive disk accesses. For example, a traditional deduplication system restoring a single file has to read file metadata first and then employs the file metadata to locate chunk metadata. Therefore, in the worse case, if the file metadata is $100 \%$ random, then restoring $\mathrm{N}$ files will generate $2 \mathrm{~N}$ disk accesses for the metadata. Figure 1 depicts the comparison of the number of disk access between our CMR and traditional recovery methods in this worse case. Because the traditional recovery method needs to retrieve the file metadata from the backup metadata each time a file is restored, it causes $\mathrm{N}-1$ more disk accesses when restoring $\mathrm{N}$ files compared to our CMR. Excessive disk access, in turn, will result in a reduction in restoring performance.

In addition to the restoring performance improvement brought by our CMR, we also increase the throughput by aggressively prefetching metadata without sacrificing the deduplication ratio. Experimental results show that our CMR is feasible and effective. Our extensive experiments also indicate that CMR outperforms other rewriting algorithms in terms of restoring performance.

The rest of the article is organized as follows: Section 2 summarizes the related work including related deduplication technologies for backup and restore. Section 3 describes the implementation 
and architecture of CMR proposed in this article. Section 4 outlines the experimental setup and discusses the experimental results. Finally, Section 5 draws conclusions along with the contributions of this research.

\section{RELATED WORK}

In computing, data deduplication has been widely used to improve storage utilization and increase network throughput by eliminating duplicate copies of repeating data. A growing number of techniques were proposed focusing on the deduplication backup process $[2,4,28]$ in which the backup stream is first divided into fixed-size blocks or variable-size blocks, and then a hash function MD5 or SHA-1 [20,22] is used to calculate the fingerprint for these data chunks. Each fingerprint acts as a unique identifier that can be used by the deduplication backup process to identify whether the current processing chunk is duplicated. As the backup process continues, the current processing chunk is compared to the stored copy to see if a match occurs. If the chunk does not exist, then write it directly to disk; otherwise, replace the redundant chunk with a reference pointing to the stored copy and discard it. The chunks written to disk are aggregated one by one into a unit called a container. A container is an operating unit that the system reads and writes, and it maintains chunk locality to enhance deduplication ratio. Unfortunately, the existing data deduplication techniques are inadequate for improving restoring performance. As the number of backup increases, the shared chunks between backups are distributed among various containers. Such a chunk distribution results in performance degradation. More specially, multiple containers must be accessed to restore the latest backup, which greatly reduces system throughput, and this negative effect is also known as fragmentation.

To improve the restoring performance of deduplication systems, an increasing number of rewriting algorithms, at the price of deduplication ratio, have been studied. We classify these algorithms into the following categories.

\subsection{Continuous Sequences Deduplication}

Srinivasan et al. [24] proposed an inline deduplication system called iDedup for primary storage. In iDedup, all relevant index data are stored in main memory. Successive repeated chunks are deduplicated only if they are long enough, and the corresponding copies of these chunks must be placed contiguously on the disks. Nam et al. developed a selective deduplication method that defines a variable called block fragmentation level, which is used to estimate the degree of fragmentation [21]. If the block fragmentation level is less than a predetermined threshold, then selective deduplication is performed on the chunk in the segment. In Nam's selective deduplication process, if there are successive sequences of duplicate chunks in the same container, the duplicate chunks in these sequences will be deleted. Unfortunately, this method does not periodically deduplicate the chunks belonging to the same container, which leads to a decrease in deduplication ratio.

\subsection{Unordered Rewriting}

Content-based rewriting algorithms [12] and container capping rewriting algorithms [15] are different from the aforementioned sequence deduplication approachs. The container capping rewriting algorithms divide backup data into chunks, and pack a number of chunks into segments by a segmentation algorithm. Each segment has a fixed size of containers and the segmentation algorithm processes one segment at a time. Capping rewriting algorithms make use of the capping level aiming to limit the number of containers that will be read to load duplicate chunks. More specifically, capping rewriting algorithms limit the container reads required to restore a segment to $\mathrm{T}+\mathrm{C}$, where $\mathrm{C}$ is the number of new containers generated during segment deduplication, and $\mathrm{T}$ denotes the capping level. Similar to Capping rewriting algorithms, to obtain a higher bound 
on the number of container reads, Content-based rewriting algorithms set a capping level for all the segments by using a fixed threshold. However, Capping-based rewriting algorithms have a limitation, they fail to make deduplication ratio as an optimization goal. There is abundant room for improvement on tradeoff between the data deduplication ratio and the number of container$\operatorname{read}[3]$.

\subsection{History-aware Rewriting}

Fu et al. [9] designed a history-aware rewriting algorithm attempting to improve the accuracy of fragment identification. Fu's findings advocate that most of the chunks in a number of containers are never accessed during recovery. These containers are called sparse containers, and sparse containers will remain sparse in the next backup. As backups increase, new sparse containers may also appear. Fu suggests that reducing sparse containers can improve recovery performance. Follow this thinking, to rewrite chunks in sparse containers, Fu defined container utilization for each container. Containers with utilization below a predefined threshold are marked as sparse containers so that it is possible to identify sparse containers after each backup. In doing so, the current backup can provide information about the sparse containers for the next backup. During the next backup, the rewrite of the sparse containers in the last backup is performed. Fu's rewriting algorithm enlarges the scope of fragment identification and uses historical information between backups to enhance the accuracy of the fragment identification.

Unlike aforementioned approaches, our metadata classifying strategy embrace parallelism for the recovery process. Parallel processing in storage system can be divided into spatial parallelism and temporal parallelism. Spatial parallelism refers to the simultaneous execution of tasks by several processing units while temporal parallelism means the simultaneous implementation of multiple operating stages. To explore the parallelism of the deduplication systems, studies have been proposed by Liu et al. [17], Guo et al. [10], and Pdedup [29]. P-dedup implements parallel chunk through a content-based block algorithm while the other two schemes rely on fixed chunk algorithms. And all steps of deduplication is performed in a parallel manner in these three methods, for example, calculating the fingerprint of one chunk while writing another chunk to disk.

\section{SYSTEM ARCHITECTURE}

A general restore process can be broken down into three stages, which are read backup metadata, find the backup data, and, finally, restore the backup. Similarly to the parallelism applied in the backup process, the restore process can make use of parallelism to improve restoring performance. Because a general restore process is separated into three stages, overlapping these three separate stages can significantly improve restore performance. Our CMR embraces a salient featureparallelism-and the reason is threefold. First, CMR judiciously classifies backup metadata into file metadata and chunk metadata. Second, CMR maintains the entire file metadata in memory to avoid on-disk metadata access for files. Third, CMR effectively reduces on-disk metadata accesses by aggressively prefetching chunk metadata into memory. In addition to CMR's parallel capability, CMR also expands deduplication rate.

To quantitatively evaluate the effectiveness of CMR, we implemented a prototype. Figure 2 depicts the architecture of CMR, which consists of the following five modules: Classifying Metadata module (CM), Radically Prefetching Metadata module (RPM), Chunk Lookup module (CL), Container Pool (CP), and Restoring Stream module (RS). The CM is responsible for separating backup metadata into file metadata and chunk metadata. The RPM module focuses on aggressively prefetching metadata. The LRU cache-based CL module provides a block lookup function that manages thermal information related to backup restoring. The RS module is responsible for reconstructing the required data on local disk. 


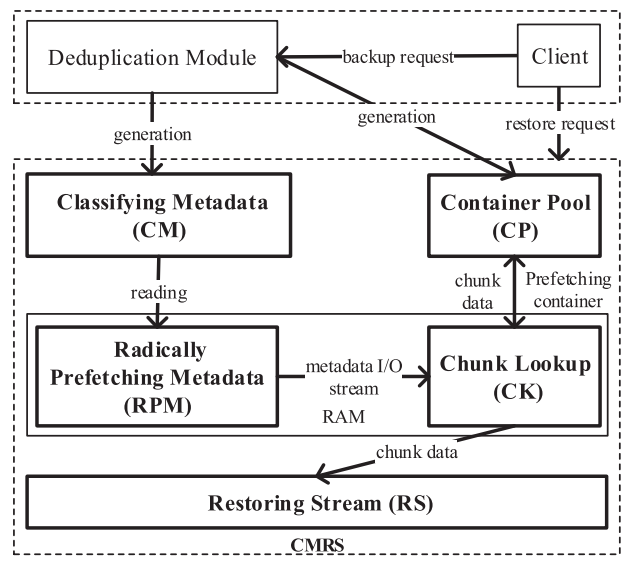

Fig. 2. System overview of CMR.

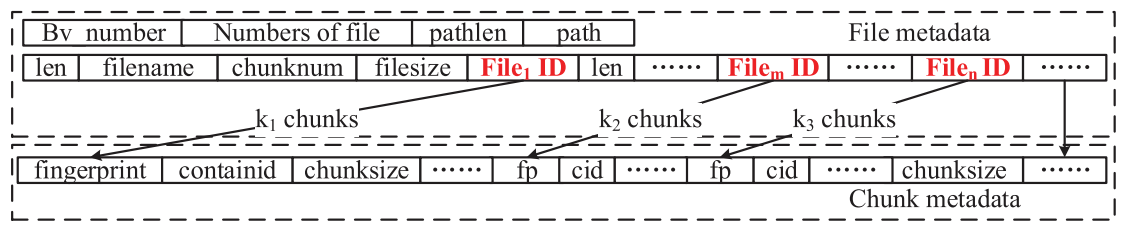

Fig. 3. Structure of classified file metadata and chunk metadata.

These five modules work in parallel with each other during a restoring process. Figure 2 also demonstrates the workflow of our system. CMR completes data recovery through the following steps:

(1) When the deduplication system is backing up, it classifies metadata into two types: file metadata and chunk metadata.

(2) By pretching the contents of the CM, RPM obtains detailed information about storage chunks, chunks' fingerprints, and chunks' location associated with backups.

(3) According to chunk's fingerprint, the CL looks for the container that contains this chunk in the maintained cache. If it hits, then the cache fills the chunk data in the memory queue. Otherwise, the CL module searches the CP for the chunk data, and the container containing the chunk is prefetched into the cache.

(4) Finally, the RS restores data streams to a specified directory.

\subsection{Classifying Metadata}

After a backup is completed, the deduplication system only generates metadata required for previous data recovery. Our CMR classifies and stores the backup metadata as two types. One is called file metadata, which record logical file information in the backup, and the other is called chunk metadata, which record information about chunks.

Figure 3 shows the classified metadata structure after a backup. The first two lines of the formation from the top are recorded as file metadata. Since a deduplication system often needs to store multiple backups, we generate a backup serial number for each backup so that the system can determine which backup needs to be restored. For each backup, file metadata (see the first line in Figure 3) includes four information: its backup serial number, the number of files that make up the backup, the path stored after the backup, and the length of the path name. Because a backup 


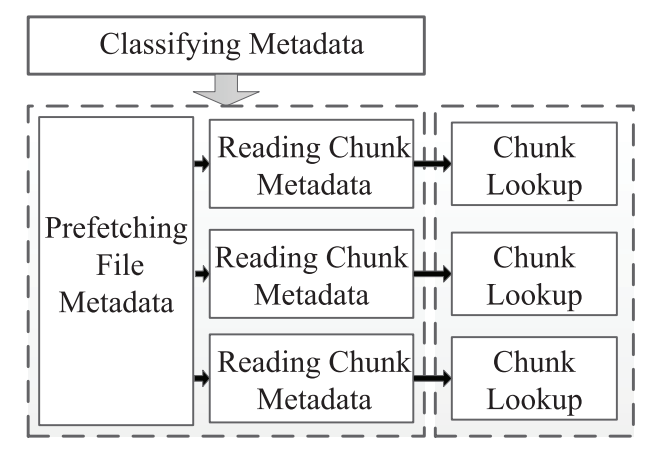

Fig. 4. Overview of radically prefetching metadata.

consists of multiple files, and each file is divided into multiple chunks, each file metadata (see the second line in Figure 3) consists of file name associated with its backup, the length of the file name, the number of files, the file size, and file ID. Moreover, a filename is stored in the absolute path format. The third row in Figure 3 is chunk metadata, each of which contains three information: a fingerprint, a container ID and its chunk size. File metadata and chuck metadata are linked, and they both guide the restoration process. Such a metadata structure enables CMR prefetch in an efficient fashion.

\subsection{Radically Prefetching Metadata}

Meister's study advocates that backup metadata takes up a certain amount of storage space. More specifically, $1 \mathrm{~TB}$ logical data with an average chunk size of $8 \mathrm{~KB}$ requires 2.5-GB storage space [18]. However, in our experiments, we observed that for backing up 6-GB data, file metadata take up $4 \mathrm{~KB}$ and chunk metadata $35 \mathrm{M}$. Now we can see that after classifying metadata into file metadata and chunk metadata, the space of file metadata is only $1 /\left(256^{*} 35\right)$ of that of chunk metadata. This is because the number of files in a backup is much smaller than the total number of file chunks. To illustrate the efficiency of file metadata in memory space saving, we make use of the following mathematical equations to calculate the size of file metadata. Assuming that the current backup contains 10 million files, then according to the metadata structure shown in Figure 3, the size of the file metadata is expressed as Equation (1), where $S_{L}, S_{F N}, S_{C N}, S_{F S}$, and $S_{I D}$ denote the len, the file name, the chunk number, the file size, and the File ID, respectively. Now, it can be calculated that in the case of 10 million files, the size of the file metadata is only about $340 \mathrm{MB}$,

$$
\begin{aligned}
S_{\text {file metadata }} & =\left(S_{L}+S_{F N}+S_{C N}+S_{F S}+S_{I D}\right) * N_{F N} \\
& =(4+8+8+8+8) * 10000000 \text { byte } \\
& \approx 343 M B .
\end{aligned}
$$

Since file metadata consume only a small amount of memory space, we propose a greedy prefetching method to leverage the memory by aggressively perfecting chunk metadata into it. Figure 4 shows the RPM. The RPM first completely prereads the file metadata to the memory and then aggressively prefetches chunk metadata when the system begins to restore. Fully prefetched file metadata residing in memory reduce the number of accesses to the disk. To strengthen reading intensity, the prefetched file metadata are divided into a set of slices, and each slice contains a file metadata, which will call a chunk metadata read operation.

To read chunk metadata in parallel, we introduce an index called File ID. Based on the File ID, each invoked read operation can locate the associated chunks. A file ID contains three parts: displacement operation associated with its backup number, an offset indicating the starting point to 


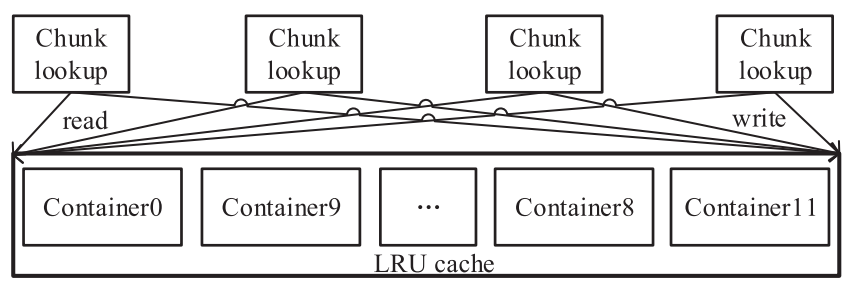

Fig. 5. The principle of cache sharing.

read, and the number of chunks contained in the file. The offset indicates the location each reading operation begins to read in the chunk metadata. Now, let us take a concrete example to illustrate the parallel chunk metadata reading. From Figure 3 we can see three highlighted File IDs in red: File ${ }_{1}$, File $_{m}$, File $_{n}$. From the File ID introduction mentioned above, we derive that restoring File requires reading the chunk metadata $\mathrm{k}_{1}$, File $\mathrm{e}_{m}$ requires reading $\mathrm{k}_{2}$ and File ${ }_{n}$ requires reading $\mathrm{k}_{3}$. Suppose these three File IDs $\mathrm{k}_{1}, \mathrm{k}_{2}$, and $\mathrm{k}_{3}$ belong to three different file slices $\mathrm{SL}_{1}, \mathrm{SL}_{m}, \mathrm{SL}_{n}$ respectively. Accordingly, three corresponding chunk metadata reads will be invoked independently by $\mathrm{k}_{1}, \mathrm{k}_{2}$, and $\mathrm{k}_{3}$, respectively. Since these three independent metadata reading operations do not interfere with each other, based on the offset File ID, required metadata at different locations can be loaded into memory simultaneously. In short, such a classifying-metadata-based chunk-prefetching method relies on the index File ID. Without File ID in place, simultaneous reading operations will result in a disordered information obtainment.

\subsection{Cache Sharing}

Chunk lookup module mainly focuses on performing chunk looking up during metadata reading. This module maintains a number of independent search operation units. Originally, we maintained an LRU cache for each operation unit, and each unit performs search operations for the required chunk data on its associated cache. Thanks to the independence of search operation units, the searching processes are executed in parallel and does not affect each other. However, we must consider the locality issue, that is, a container stored in its cache processed by its unit may involve other containers stored in other caches. To improve the searching performance, instead of having multiple individual LRU caches, we finally store all the containers within a single global LRU cache in the memory, so that containers can be accessed and processed by multiple units simultaneously (see Figure 5).

As we can see in Figure 5, all operating units access a shared cache, so that they can take full advantage of the locality in a data stream. More specifically, a container that a unit inserts into the shared cache also provides chunks for other units. Such a shared cache not only improves the cache hit rate but also reduces the number of disk accesses, thereby reducing the time cost. However, multiple operating units may perform write operations on the shared cache. When a unit needs to insert a container into the shared cache, it needs to kick out a container. In this scenario, we facilitate a read-write lock to ensure the integrity of shared data. Specifically, when the current operating unit is writing on the shared cache, the cache is locked, and other units are not be able to modify the cache until the shared cache is unlocked by the current unit.

\subsection{Restoring Stream}

After the required chunk data are found, the system starts restoring the required data stream. The restoring stream module has multiple data writing units, and each data writing unit is responsible for restoring several files. Since the filenames specified by the restore quests are in an absolute path format (see Figure 3) and each data writing unit creates its own file directory, there 
is no chance for data writing units to create file directories with the same name. Therefore, file restoring operations performed by different data writing units will not interfere with each other. Restoring stream module carries out restoring tasks simultaneously through multiple threads, and each thread undertake one restoring task at a time. This parallelism implementation even enable data writing units restoring multiple files in the same directory in parallel. In doing so, a writing unit can perform a restore operation even before other units complete their restore operations, thereby avoiding additional computing overhead.

\subsection{Parallel Restore}

Traditional recovery processes perform operations in sequence as the following three steps: (1) read backup metadata, (2) find backup data, and (3) restore backup. Within a multi-processor system, it is possible to improve restoring performance through the multi-processor parallelism. Moreover, to further boost restoring performance, in our design, each recovery-process running on a single pipelined-processor is multithreaded. More specially, in our recovery process, the deduplication system first prefetches file metadata into memory and divides the file metadata into multiple slices. Then, it assigns each slice to a thread within a pipelined-process running on a single processor. In doing so, the throughput of a recovery process is boosted up to multiple files rather than one. Sine File metadata is divided into a set of slices and each slice is handled by a thread. To meet computing needs, we dynamically adjust the number of pipelines as well as the number of pipelined-processes according to the number of slices. As a result, the more slices are divided, the more threads are assigned, and the higher performance we achieve.

\section{PERFORMANCE EVALUATION}

\subsection{Experimental Environment}

To quantitatively evaluate the restoring performance of our classified-metadata-based CMR approach, we compare CMR with two state-of-the-art algorithms: container capping rewriting algorithm (CAP) [15] and history-aware rewrite algorithm (HAR) [9]. In addition, a traditional backup metadata organization method (destor (DR)) is used as the experimental baseline scheme. We conduct all the experiments on a server, whose hardware configuration is as following: a dual-core, four-thread Intel Core i3-2100 3.10-GHz processor, a 10-GB memory, and a 500-G disk drive and the operating system is Ubuntu (kernel version 3.16.0).

For fair comparison purpose, we apply two real datasets for running the experiments. Dataset 1 is a commonly used Linux kernel source code collection downloaded from the Internet. This dataset contains 176 versions, and each version consists of a handful small files. Since the average size of these 176 versions is around $530 \mathrm{MB}$, the total size of Dataset 1 is $95 \mathrm{~GB}$. Dataset 2, as virtual machine disk image files, is collected from a working directory by our laboratory members. Dataset 2 contains only 81 files for a total of 71 GB. We apply different backup strategies on these two datasets. On Dataset 1 we adopt full backup, the backup number is set to 8 and each backup contains various versions of the kernel source code. The size of the last backup equals to the total size of the Dataset 1. Dataset 2 is composed of eight backups, each with a slightly different size. Table 1 summarizes the characteristics of these two datasets. It can be seen from Table 1 that Dataset 1 is quite different from Dataset 2, especially in the number of files. Dataset 1 represents a collection of small files, whereas, Dataset 2 represents a collection of large files.

\subsection{Determining the Optimal Number of Slices}

As we discussed in Section 3.2, CMR restoring process first prefetches all the file metadata into memory, divides the file metadata into several slices, and then assigns each slice to a thread. The 
Table 1. The Characteristic of Datsets

\begin{tabular}{|c|c|c|c|c|}
\hline & \multicolumn{2}{|c|}{ Dataset 1 } & \multicolumn{2}{c|}{ Dataset 2 } \\
\hline Rank & Size & Numbers & Size & Numbers \\
\hline backup0 & $13 \mathrm{~GB}$ & 1145602 & $6 \mathrm{~GB}$ & 10 \\
\hline backup1 & $28 \mathrm{~GB}$ & 2456790 & $6 \mathrm{~GB}$ & 10 \\
\hline backup2 & $38 \mathrm{~GB}$ & 3350104 & $5 \mathrm{~GB}$ & 10 \\
\hline backup3 & $52 \mathrm{~GB}$ & 4590511 & $6 \mathrm{~GB}$ & 10 \\
\hline backup4 & $65 \mathrm{~GB}$ & 5672392 & $11 \mathrm{~GB}$ & 12 \\
\hline backup5 & $77 \mathrm{~GB}$ & 6791898 & $9 \mathrm{~GB}$ & 10 \\
\hline backup6 & $86 \mathrm{~GB}$ & 7610448 & $11 \mathrm{~GB}$ & 10 \\
\hline backup7 & $95 \mathrm{~GB}$ & 8366722 & $17 \mathrm{~GB}$ & 9 \\
\hline
\end{tabular}

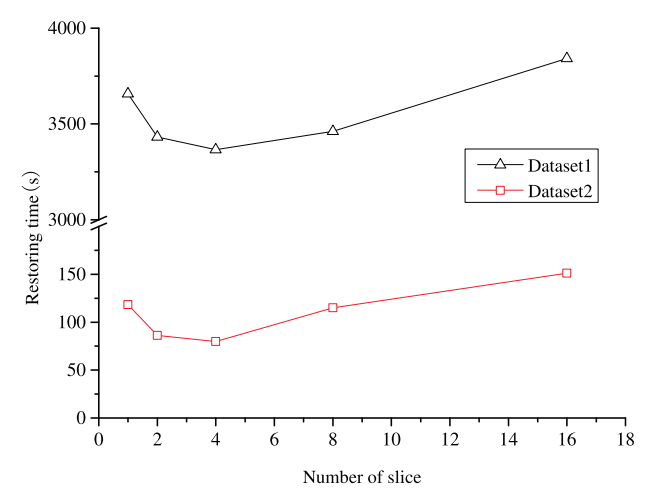

Fig. 6. Restoring time with different number of slices.

segmentation method utilized by CMR to divide the file metadata has a significant impact on restoring performance. The slice mechanism allowing CMR to greedily prefetch the chunk metadata in parallel boosts the restoring performance.

However, such a parallel method must determine the optimal number of launched threads for minimizing the thread overhead and locking overhead. The more threads created, the more overhead caused by CMR. More specifically, pipelines need to access the shared memory. To ensure data consistency, locks are introduced so that multiple waiting processes compete for certain locks for exclusively working on the assigned file metadata slices. These two effects give rise to an additional operational overhead.

To determine the optimal number of slices, we conduct a set of experiments to profile the impact of the number of slices on performance. In this part of the study, we carry out performance profiling on two datasets: backup 7 of Dataset 1 and the backup 3 of Dataset 2, respectively. We vary the number of slices from 1 to 16 . The cache is set to consist of 1,024 containers for restoring these two datasets. Figure 6 shows the restoring time as a function of the number of slices. The profiling results reveal the optimal number of divided slices leads to the highest restoring performance. Specifically, in Dataset 1 case, when the number of slices is 4 , the restoring performance reaches the highest level. Figure 6 also shows that the restoring performance of the 4 slices case is 1.09 times of that of the 1 slice case. Such a performance boost is attributed to the benefits brought by parallelism. However, as number of slices increases from 4 to 16, the restoring performance degrades. The restoring time of the 4 slices case is $1.14 \times$ than that of the 16 slices case. This is 


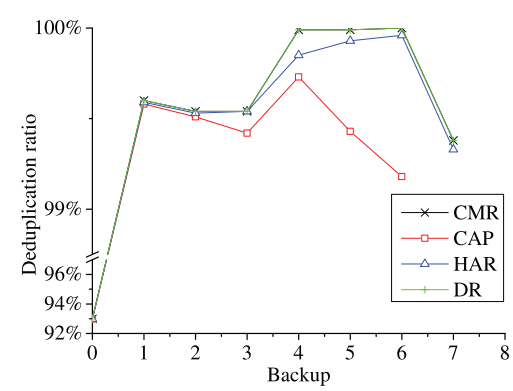

(a) Dataset1

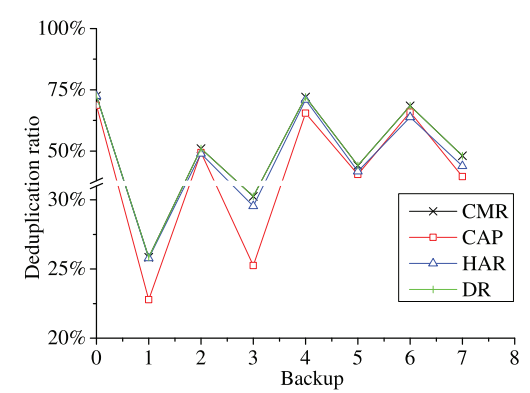

(b) Dataset2

Fig. 7. Deduplication ratio of eight backups with CMR, CAP, HAR, and DR.

because these 16 slices cost higher overhead than 4 slices, which hurts the benefits of parallelism. The curves plotted in Figure 6 on Dataset 2 show a similar trend to that on Dataset 1, and the best restoring performance is obtained when the number of slices is 4 . To demonstrate the rationality of the experiments, we adopt the middle value strategy-we set the default number of slices to 2 , if there is no special note.

We performed experiments by increasing the memory size and reducing the volume of datasets. Although the memory utilization and CPU utilization are decreased, the optimal number of slices for file metadata is still four as Figure 6 demonstrates. This is because each slice is used by a thread to perform parallel restoring, and our experiments are performed with a dual-core, four-thread Intel processor. Therefore, four slices can fully leverage the physical parallelism of processor.

\subsection{Evaluating the Deduplication Ratio}

Deduplication ratio is the ratio of deduplication data size to the original data size. Most existing rewriting algorithms improve restoring performance at the expense of deduplication ratio. Because a modern data center is in pressing demand of backing up an increasingly massive amount of data, such massive data takes up huge disk space. Striving to save the disk space, we propose CMR method that does not use a rewriting algorithm during data backup.

We conduct a group of experiments to evaluate the deduplication ratio saving of our CMR. This group of experiments is performed on the eight different backups that come from two datasets (Dateset 1 and Dataset 2). Unlike traditional backup processes that rely on rewriting algorithms, we leverage Rabin chunk-a classic variable content chunk algorithm for data backup. The average chunk size is $4 \mathrm{~KB}$ and the index cache size is set to 128 containers.

Now, we compare the backup deduplication ratio of our CMR with that of other three schemes, namely, CAP, HAR, and DR. And CAP uses a preset threshold size of 10, HAR uses a predetermined utilization threshold of $50 \%$. We increase the number of backups from 1 to 8 . In Figure 7, the $X$ axis represents eight successive full backups of the dataset, starting from the leftmost backup 0 to the rightmost backup 7. Figure 7(a) shows that when using Dataset 1, the deduplication ratio of all backups is very high. For example, the deduplication ratio of CMR, CAP, and HAR is $98.88 \%$, 98.49\%, and $98.83 \%$, respectively. Figure 7(a) also reveals that although CMR, CAP and HAR perform similarly in terms of deduplication ratio, our CMR outperforms CAP and HAR. For example, CMR rewrites $0.04 \%$ more data than HAR does for backup 6. Although this is a slim difference in terms of deduplication ratio, when coming to the perspective of disk space, it turns out to a huge difference. Specifically, the size of backup 6 is 86 G (see Table 1), we can derive that for backup 7, compared with CMR, HAR wastes 36-M disk space, while CAP wastes $1.3 \mathrm{G}$. 


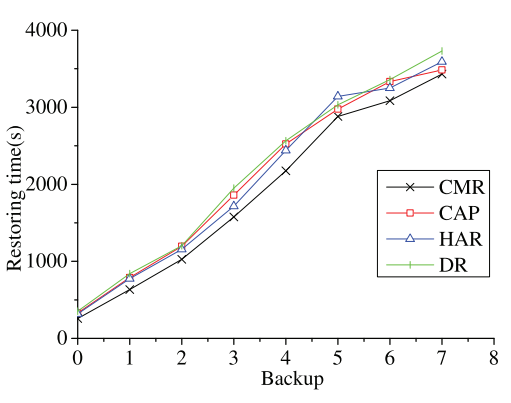

(a) Dataset1

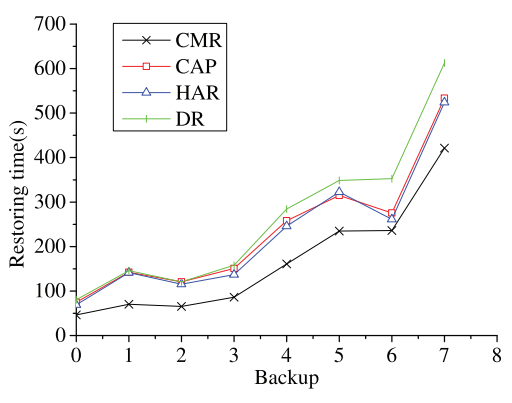

(b) Dataset2

Fig. 8. Restoring time of eight backups with CMR, CAP, HAR, and DR.

Moreover, Figure 7(a) shows that as the number of backup increases, the deduplication ratio difference between our CMR and CAP becomes larger. This is because as the number of backups increases, fragmentation impact becomes more severe [15]. Due to fragmentation, CAP must rewrite more block data. When the number of backups reaches a turning point, CAP's deduplication ratio starts to decline. Unlike CAP, CMR does not use rewriting algorithms to save disk space when backing up data, thereby, avoiding such a negative fragmentation impact. HAR is also superior to CAP in terms of deduplication ratio, the reason behind is that CAP identify sparse containers in a limited-size buffer, it is possible for CAP to rewrite chunk data belonging to an out-of-order container [9], whereas HAR makes use of links between backups and identified sparse containers, which reduces unnecessary rewriting.

Figure $7(\mathrm{~b})$ indicates that, for Dataset 2, the average deduplication ratio of CMR, HAR, and CAP is $51.54 \%, 49.63 \%$, and $47.18 \%$, respectively. And the deduplication ratio descends as the number of backups increases. Our CMR saves an average of $1.91 \%$ and $4.36 \%$ deduplication ratio compared with HAR and CAP, respectively. Figure 7(b) also reveals that without using rewriting algorithms, the deduplication ratio of CAP and HAR becomes relatively low. For example, CAP and HAR have a similar deduplication ratio for backup 1 and backup 3, and this ratio is approximately $25 \%$ and $30 \%$ in the backup 1 and backup 3 cases, respectively. Since our CMR does not rely on rewriting algorithms, it does not suffer such a deduplication ratio drop. For example, in the backup 7 case, the deduplication ratio of CMR is $25 \%$ higher than that of CAP, and $12.8 \%$ higher than that of HAR.

\subsection{Evaluating the Restoring Performance}

Prior algorithms improve restoring performance at the cost of the deduplication ratio. To address this sacrifice issue, we propose CMR, which improves restoring performance without deduplication ratio reduction. To evaluate the restoring performance of CMR, we conduct a group of experiments. We set the default buffer size to 1,024 containers and the CMR recovery process divides file metadata into two slices, as described in Section 4.2.

Figure 8 shows the restoring times for Dataset 1 and 2 when four algorithms are applied, namely, CMR, CAP, HAR, and DR. The four curves plotted in Figure 8(a) exhibit an upward trend, that is, as the number of backups goes up, the restoring time increases. This upward trend of the restoring time is expected, because more fragments are generated as the number of backup increases, which in turn, hurts the recovery performance. Figure 8(a) indicates that CMR requires less restoring time than other three methods. DR has the highest average restoring time (i.e., the worst performance). Take the backup 4 case as an example, the restoring time of CMR is $2174.365 \mathrm{~s}$, and DR costs $2564.818 \mathrm{~s}$, hence, DR is $1.18 \times$ slower than our CMR. The reason is twofold: First, DR does not greedily prefetch chunk metadata in the recovery process, and, second, DR takes advantage of 


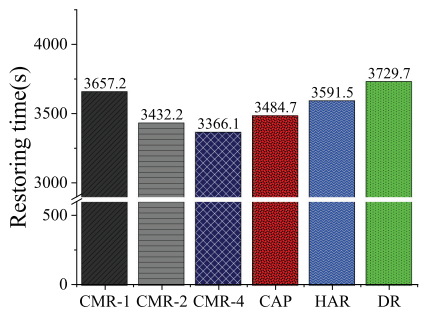

(a) Dataset 1

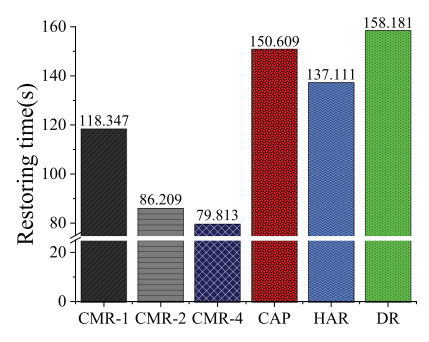

(b) Dataset 2

Fig. 9. Restoring time of CMR with different threads, CAP, HAR, and DR.

only one pipeline. In short, CMR not only leverages multiple pipelines but also prefetches the entire file metadata.

Figure 8(a) shows that, for backup 3, the restoring time of HAR, CAP, and DR is $1714.583 \mathrm{~s}$, $1859.234 \mathrm{~s}$, and $1951.153 \mathrm{~s}$, respectively. The average restoring time of HAR and CAP is less than that of DR, this is because the rewriting algorithm utilized by HAR and CAP effectively defragments the fragmentation so that the recovery process reduces the number of accesses to the disk. The restoring time consumed by CMR is always less than that by HAR and CAP (see Figure 8(a)), this confirms that our proposed restoring scheme outperforms other three schemes in terms of the restoration performance. Combined with former experimental results discussed in Section 4.3, we can conclude that CMR achieves the desired restoration performance without losing deduplication ratio.

Unlike Figure 8(a), which depicts the restoring performance for small files, Figure 8(b) shows the restoring performance for large files. It demonstrates that CMR has the least restore time, averaging $165.203 \mathrm{~s}$ in eight backups. Whereas, the average restoring time of HAR, CAP, and DR is $227.033 \mathrm{~s}, 233.684 \mathrm{~s}$, and $262.976 \mathrm{~s}$, respectively. CMR reduces the average restoring time of HAR and CAP by $27.2 \%$ and $29.3 \%$, respectively. It is noteworthy that in these groups of experiments, CMR simply divides the file metadata into two slices to restore backup data. Since the more slices are divided, the more associated threads present, and the higher performance we achieve (see detailed discussion in Section 4.2). If we increase the number of divided slices of the file metadata, then a further remarkable performance boost will be achieved.

To investigate the performance impact of parallelism on the restoring time, we compare CMR against HAR, CAP, and DR, we vary the number of threads of CMR to one thread, two threads, and four threads, respectively. To be consistent with Figure 6, we carry out the parallelism performance profiling by using the backup 7 of Dataset 1 and the backup 3 of Dataset 2, respectively. Figure 9 shows the restoring time of CMR, CAP, HAR, and DR, where CMR-1, CMR-2, and CMR-4 represent CMR approach with one thread, two threads, and four threads, respectively. It demonstrates that when using Dataset 1, the resotring performance of CMR-1 outperforms that of DR approach. However, it is worse than that of CAP and HAR. After improving the parallelism by increasing the number of threads, the restoring time of CMR-2 outperforms that of CAP and HAR, and CMR-4 achieves the best restoring performance as expected.

It is noteworthy that when using Dataset 1 , only in the CMR-1 case (i.e., only one tread is created for prefetching chunk metadata), the restoring performance of CMR is worse than that of CAP and HAR. This is expected. As discussed in Section 4.1, Dataset 1 consists of small files, the deduplication ratio of Dataset 1 is much higher than that of Dataset 2, and the disk fragmentation becomes severe with the increase of the deduplication ratio. Both CAP and HAR are overwriting-based 


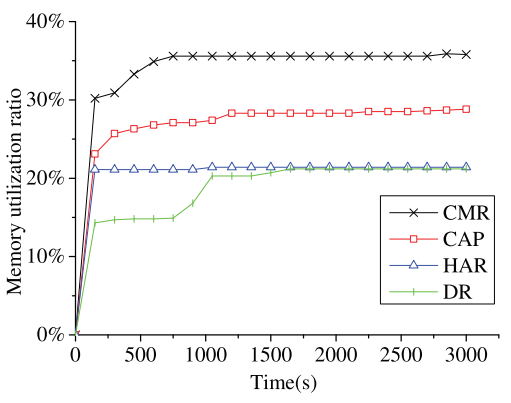

(a) Dataset1

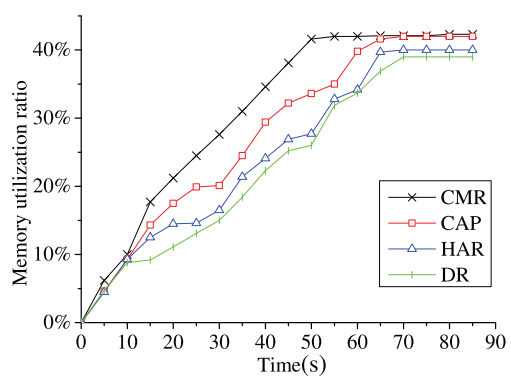

(b) Dataset2

Fig. 10. Memory utilization ratio when using CMR, CAP, HAR, and DR with dataset 1 and dataset2.

Table 2. Average CPU Utilization When Using CMR, CAP, HAR, and DR with Dataset 1 and Dataset 2

\begin{tabular}{|c|c|c|}
\hline Average CPU utilization & Dataset 1 & Dataset 2 \\
\hline CMR & $37 \%$ & $33.67 \%$ \\
CAP & $27.25 \%$ & $16.67 \%$ \\
HAR & $22.5 \%$ & $18.67 \%$ \\
DR & $24.25 \%$ & $7.67 \%$ \\
\hline
\end{tabular}

methods dedicating to alleviate fragmentation. However, as more chunk metadata prefecthing threads are launched, CMR can easily outperform CAP and HAR in terms of restoring performance. Moreover, when using Dataset 2, the restoring performance of CMR-1 is substantially better than both CAP and HAR. This is because in contrast to Dataset 1, Dataset 2 consists of virtual machine disk image files, which are all big files. These big files do not cause significant disk fragmentation.

\subsection{Evaluating the System Overhead}

In addition to the evaluation of the restoring performance discussed above, in this set of experiments, we focus on evaluating the system overhead caused by the four recovery strategies (CMR, CAP, HAR, and DR). We measure and record the memory and CPU utilization of these four schemes on Dataset 1 and Dataset 2, respectively. As we can see in Figure 10 that our CMR restoring strategy causes more memory overhead than the two rewriting algorithms do (CAP and HAR). Such an additional memory overhead is expected, because with CMR in place, after prefetching the total file metadata into the memory, the memory system needs to accommodate the multi-threaded executions incurred by the divided-file-metadata slices. Moreover, this corresponds to our original intention-leveraging memory thoroughly to improve the restoring performance. Table 2 summarizes the average CPU utilization of the four restoring strategies (CMR, CAP, HAR, and DR) on Dataset 1 and Dataset 2, respectively. Compared with the other three methods, CMR exhibits higher CPU utilization on average, however it does not take up disproportionate memory resource. (Table 2.)

Unlike fingerprint calculations during deduplication, data recovery process is not computationally intensive. Therefore, we can conclude that most of the times in a CMR recovery process, the $\mathrm{CPU}$ is allocated to the existing worker threads. 


\section{CONCLUSION}

In this article, we propose a classified-metadata-based restoring system called $C M R$. We judiciously classify backup metadata into file metadata and chunk metadata. The total file metadata is prefetched to memory to reduce the number of disk reads. When free space of memory is sufficient, we aggressively prefetch chunk metadata to memory in a greedy manner. The file metadata is divide into slices, and each slice is assigned to a thread for processing. Such a parallel restoration approach leverages computing resources. Unlike traditional restoring strategies, our CMR does not rely on rewriting algorithms. Thus, CMR improves restoring performance without deduplication ratio loss. Experimental results show that CMR improves the restoring performance of the existing schemes by approximately $30 \%$.

We believe that the restoring performance of CMR can be further improved with the growth of the system scale. Fragmentation caused by traditional deduplication techniques can easily offset the benefits of multiprocessor parallelism. To address this issue, as a future research direction, we plan to exploit computing nodes to optimize the restoring process and improve the restoring performance.

\section{REFERENCES}

[1] Deepavali Bhagwat, Kave Eshghi, Darrell D. E. Long, and Mark Lillibridge. 2009. Extreme binning: Scalable, parallel deduplication for chunk-based file backup. In Proceedings of the IEEE International Symposium on Modeling, Analysis \& Simulation of Computer and Telecommunication Systems (MASCOTS'09). IEEE, 1-9.

[2] Pramod Bhatotia, Rodrigo Rodrigues, and Akshat Verma. 2012. Shredder: GPU-accelerated incremental storage and computation. In Proceedings of the USENIX Conference on File and Storage Technologies (FAST'12).

[3] Zhichao Cao, Shiyong Liu, Fenggang Wu, Guohua Wang, Bingzhe Li, and David H. C. Du. 2019. Sliding look-back window assisted data chunk rewriting for improving deduplication restore performance. In Proceedings of the 17th USENIX Conference on File and Storage Technologies (FAST'19). 129-142.

[4] Biplob Debnath, Sudipta Sengupta, and Jin Li. 2010. ChunkStash: Speeding up inline storage deduplication using flash memory. In Proceedings of the 2010 USENIX Conference on USENIX Annual Technical Conference (ATC'10).

[5] Yuhui Deng. 2011. What is the future of disk drives, death or rebirth? Comput. Surv. 43, 3 (2011), 1-28.

[6] Yuhui Deng, Xinyu Huang, Liangshan Song, Yongtao Zhou, and Frank Wang. 2017. Memory deduplication: An effective approach to improve the memory system. F. Inf. Sci. Eng. 33, 5 (2017), 1103-1120.

[7] Yuhui Deng and Frank Wang. 2008. Exploring the performance impact of stripe size on network attached storage systems. J. Syst. Arch. 54, 8 (2008), 787-796.

[8] Kave Eshghi, Mark Lillibridge, Lawrence Wilcock, Guillaume Belrose, and Rycharde Hawkes. 2007. Jumbo store: Providing efficient incremental upload and versioning for a utility rendering service. In Proceedings of the USENIX Conference on File and Storage Technologies (FAST'07). 123-138.

[9] Min Fu, Dan Feng, Yu Hua, Xubin He, Zuoning Chen, Wen Xia, Fangting Huang, and Qing Liu. 2014. Accelerating restore and garbage collection in deduplication-based backup systems via exploiting historical information. In Proceedings of the 2014 USENIX Conference on USENIX Annual Technical Conference (USENIX ATC 14). USENIX Association, 181-192.

[10] Fanglu Guo and Petros Efstathopoulos. 2011. Building a high-performance deduplication system. In Proceedings of the USENIX Conference on USENIX Annual Technical Conference (ATC'11).

[11] James Hamilton. 2009. The cost of latency. Perspectives Blog. https://perspectives.mvdirona.com/2009/10/the-cost-oflatency/.

[12] Michal Kaczmarczyk, Marcin Barczynski, Wojciech Kilian, and Cezary Dubnicki. 2012. Reducing impact of data fragmentation caused by in-line deduplication. In Proceedings of the 5th Annual International Systems and Storage Conference. ACM, 15.

[13] Ron Kohavi, Randal M. Henne, and Dan Sommerfield. 2007. Practical guide to controlled experiments on the web: Listen to your customers not to the hippo. In Proceedings of the 13th ACM SIGKDD International Conference on Knowledge Discovery and Data Mining. ACM, 959-967.

[14] Erik Kruus, Cristian Ungureanu, and Cezary Dubnicki. 2010. Bimodal content defined chunking forbackup streams. In Proceedings of the USENIX Conference on File and Storage Technologies (FAST'10). 239-252.

[15] Mark Lillibridge, Kave Eshghi, and Deepavali Bhagwat. 2013. Improving restore speed for backup systems that use inline chunk-based deduplication. In Proceedings of the 2013 USENIX Conference on USENIX Annual Technical Conference (ATC'13). 183-198. 
[16] Mark Lillibridge, Kave Eshghi, Deepavali Bhagwat, Vinay Deolalikar, Greg Trezis, and Peter Camble. 2009. Sparse indexing: Large scale, inline deduplication using sampling and locality. In Proceedings of the USENIX Conference on File and Storage Technologies (FAST'09), Vol. 9. 111-123.

[17] Chuanyi Liu, Yibo Xue, Dapeng Ju, and Dongsheng Wang. 2009. A novel optimization method to improve deduplication storage system performance. In Proceedings of the 15th International Conference on Parallel and Distributed Systems (ICPADS'09). IEEE, 228-235.

[18] Dirk Meister, André Brinkmann, and Tim Süß. 2013. File recipe compression in data deduplication systems. In Proceedings of the USENIX Conference on File and Storage Technologies (FAST'13). 175-182.

[19] Hao Meng, Weizhe Zhang, Yiming Wang, Dong Li, Wen Xia, Hao Wang, and Chen Lou. 2019. Multi-parameter performance modeling based on machine learning with basic block features. In Proceedings of the 17th IEEE International Symposium on Parallel and Distributed Processing with Applications. IEEE, 316-323.

[20] Athicha Muthitacharoen, Benjie Chen, and David Mazières. 2001. A low-bandwidth network file system. Acm Sigops Operating Systems Review 35, 5 (2001), 174-187.

[21] Young Jin Nam, Dongchul Park, and David H. C. Du. 2012. Assuring demanded read performance of data deduplication storage with backup datasets. In Proceedings of the 20th International Symposium on Modeling, Analysis \& Simulation of Computer and Telecommunication Systems (MASCOTS). IEEE, 201-208.

[22] Sean Quinlan and Sean Dorward. 2002. Venti: A new approach to archival storage. In Proceedings of the USENIX Conference on File and Storage Technologies (ATC). 89-101.

[23] R. Schulman. 2004. Disaster recovery issues and solutions. Hitachi data systems white paper. Hitachi Data Systems White Paper (2004).

[24] Kiran Srinivasan, Timothy Bisson, Garth R. Goodson, and Kaladhar Voruganti. 2012. iDedup: latency-aware, inline data deduplication for primary storage. In Proceedings of the USENIX Conference on File and Storage Technologies (FAST), Vol. 12. 1-14.

[25] PRESTON W. C. 2010. Restoring deduped data in deduplication systems. http://searchdatabackup.techtarget.com/ feature/Restoringdeduped-data-in-deduplication-systems.

[26] Zhuowei Wang, Lianglun Cheng, Hao Wang, Wuqing Zhao, and Xiaoyu Song. 2019. Energy optimization by software prefetching for task granularity in GPU-based embedded systems. IEEE Transactions on Industrial Electronics 67, 6 (2019), 5120-5131.

[27] Chunlin Wu, Xingqin Lin, Daren Yu, Wei Xu, and Luoqing Li. 2014. End-to-end delay minimization for scientific workflows in clouds under budget constraint. IEEE Transaction on Cloud Computing 3, 2 (2014), 169-181.

[28] Wen Xia, Hong Jiang, Dan Feng, and Yu Hua. 2011. SiLo: A similarity-locality based near-exact deduplication scheme with low RAM overhead and high throughput. In Proceedings of the 2011 USENIX Conference on USENIX Annual Technical Conference (ATC). 26-28.

[29] Wen Xia, Hong Jiang, Dan Feng, Lei Tian, Min Fu, and Zhongtao Wang. 2012. P-dedupe: Exploiting parallelism in data deduplication system. In Proceedings of the 7th International Conference on Networking, Architecture and Storage (NAS). IEEE, 338-347.

[30] Yongtao Zhou, Yuhui Deng, Junjie Xie, and Laurence Yang. 2018. EPAS: A sampling based similarity identification algorithm for the cloud. IEEE Transaction on Cloud Computing, 6 (2018), 720-733.

[31] Benjamin Zhu, Kai Li, and Hugo Patterson. 2008. Avoiding the disk bottleneck in the data domain deduplication file system. In Proceedings of the 6th USENIX Conference on File and Storage Technologies (FAST).

Received April 2020; revised September 2020; accepted November 2020 